\title{
Identification of gene signatures and potential therapeutic targets for acquired chemotherapy resistance in gastric cancer patients
}

\author{
Jie Sun ${ }^{1 \wedge}$, Jingjing Zhao ${ }^{1}$, Zhenkun Yang ${ }^{1}$, Zhiyi Zhou ${ }^{2}$, Peihua $\mathrm{Lu}^{3 \wedge}$ \\ ${ }^{1}$ Center of Clinical Research, Wuxi People's Hospital of Nanjing Medical University, Wuxi, China; ${ }^{2}$ Department of Pathology, Wuxi People's \\ Hospital of Nanjing Medical University, Wuxi, China; ${ }^{3}$ Department of Medical Oncology, Wuxi People's Hospital of Nanjing Medical University, \\ Wuxi, China \\ Contributions: (I) Conception and design: J Sun, P Lu; (II) Administrative support: P Lu; (III) Provision of study materials or patients: J Zhao, Z \\ Zhou; (IV) Collection and assembly of data: J Sun, Z Yang, Z Zhou; (V) Data analysis and interpretation: J Sun, J Zhao; (VI) Manuscript writing: All \\ authors; (VII) Final approval of manuscript: All authors. \\ Correspondence to: Peihua Lu. Department of Medical Oncology, Wuxi People's Hospital of Nanjing Medical University, Wuxi, China. \\ Email: 1phty1_1@163.com.
}

Background: Gastric cancer (GC) is the most common type of gastrointestinal cancer, and has been studied extensively. However, resistance to chemotherapeutic agents has become a major problem, leading to treatment failure. This study aimed to investigate the molecular mechanisms mediating acquired resistance to cisplatin and fluorouracil (CF) combination-based chemotherapy in GC patients.

Methods: The microarray datasets (GSE14209, GSE30070) were downloaded from the Gene Expression Omnibus (GEO) database to identify differentially expressed genes (DEGs) and differentially expressed miRNAs (DEMs) using the limma package in R/Bioconductor. Possible targets of the DEMs were predicted using miRWalk, and the putative miRNA-mRNA regulatory network was constructed using Cytoscape software. Gene Ontology (GO), Kyoto Encyclopedia of Genes and Genomes (KEGG), and proteinprotein interaction (PPI) analyses were then conducted and visualized using the Search Tool for Retrieval of Interacting Genes (STRING) and Cytoscape. The prognostic value of hub genes was revealed by KaplanMeier Plotter. The causal relationships and interactions between proteins were displayed using DisNor. Finally, similarity analysis was conducted using the Connectivity Map (CMap) profiles to predict a group of small molecules in GC treatment.

Results: A total of 394 DEGs and 31 DEMs were identified after analysis of pre- and post-treatment samples of clinical responders to CF therapy. TM9SF4, hsa-miR-185-5p, and hsa-miR-145-5p were found to be critical in the miRNA-mRNA regulatory network. The DEGs were found to be mainly enriched in the processes of ribonucleoprotein complex assembly, catalytic activity acting on RNA, mitochondrial matrix, and thermogenesis. The DEMs were predominantly found to be involved in single-stranded RNA binding and endoplasmic reticulum lumen. HDAC5, DDX17, ILF3, and SDHC were identified as hub genes in the PPI network. Of these, HDAC5, DDX17, and ILF3 were found to be closely related to the overall survival of GC patients. DisNor identified the first neighbors of the key genes. Furthermore, CMap profiles predicted a group of small molecules, including several histone deacetylase inhibitors (HDACIs), menadione, and mibefradil, which could serve as promising therapeutic agents to reverse acquired resistance to $\mathrm{CF}$ therapy.

Conclusions: Our findings reveal new targets and alternative therapies to overcome the acquired resistance of GC patients to CF treatment.

Keywords: Gastric cancer (GC); acquired resistance; candidate small molecules; bioinformatics analysis

^ ORCID: Jie Sun, 0000-0002-5025-2391; Peihua Lu, 0000-0002-4622-3061. 
Submitted Jan 11, 2021. Accepted for publication Apr 04, 2021.

doi: 10.21037/jgo-21-81

View this article at: http://dx.doi.org/10.21037/jgo-21-81

\section{Introduction}

Gastric cancer (GC) remains the fifth most frequent type of cancer and the third leading cause of cancer-related death worldwide. More than 1,000,000 new GC cases and nearly 787,000 GC-caused deaths have been estimated for 2018 (1). Despite recent advances in cancer diagnosis and therapeutic methods, including surgery, chemotherapy, radiotherapy, and targeted therapy, the prognosis of GC patients remains poor, with a five-year overall survival (OS) below $20 \%(2,3)$. Treatment using a combination of cisplatin and fluorouracil (CF) is one of the gold-standard chemotherapies for advanced GC patients (4). However, the clinical benefits of cisplatin-based chemotherapy have been limited due to drug resistance, either intrinsic or acquired. Patients who initially respond to treatment eventually develop drug resistance, termed acquired resistance, which is emerging as a significant challenge for anticancer treatments. Cancer cells develop drug resistance through a variety of mechanisms that have not been fully established. Gene differentially expressed between the chemoresponsive state and chemoresistant state were identified as the "acquired resistance gene signatures". Therefore, not only is there an urgent need to identify alterations in genes that occur when tumors become drug resistant, there also exists a great challenge in overcoming this acquired resistance to chemotherapy. Furthermore, it is imperative to search for candidate drugs that can improve the clinical treatment of GC based on the investigation of drug-gene interactions.

In the past decade, microarray and high-throughput sequencing technologies have produced massive amounts of transcriptomic data $(5,6)$. Additionally, there are lots of shared public data platforms that allow for in-depth bioinformatics-based prediction of new candidate genes. Gene Expression Omnibus (GEO) is a free international public database that contains microarray and highthroughput functional genomics datasets that can be used as powerful tools to determine key genes and pathways in the pathogenesis and progression of tumors. Another public database, Connectivity Map (CMap), is a large-scale collection of gene-expression signatures that aids in the discovery of small molecular compounds with potential use in the treatment of specific diseases $(7,8)$. Previous studies have explored the possibility of identification of small molecule drugs that could serve as plausible anticancer agents on the basis of bioinformatics approaches $(9,10)$.

In this study, we selected the mRNA expression dataset GSE14209 and the miRNA expression dataset GSE30070 from the GEO database to identify differential expression genes (DEGs) and differential expression miRNAs (DEMs) associated with acquired resistance to CF treatment in GC patients. First, the target genes regulated by miRNAs were predicted, then the potential miRNA-mRNA regulatory network regulating CF resistance was established. Gene Ontology (GO) and Kyoto Encyclopedia of Genes and Genomes (KEGG) were performed to shed light on the biological functions of these DEGs and DEMs. We then performed protein-protein interaction (PPI) network and module analysis to identify hub genes involved in CF chemoresistance. Hub genes were validated, followed by prediction of their prognostic value. Subsequently, we also identified candidate drugs for GC by carrying out similarity analysis of CMap profiles. All these findings offer new insights into the prospective applications of novel drugs for overcoming chemoresistance in GC patients. We present the following article in accordance with the MDAR reporting checklist (available at http://dx.doi.org/10.21037/ jgo-21-81).

\section{Methods}

\section{Microarray data}

The mRNA expression microarray dataset GSE14209 and the miRNA expression microarray dataset GSE30070 were downloaded from the GEO database (http://www.ncbi. nlm.nih.gov/geo/). GSE14209 was based on the GPL571 platform [(HG-U133A_2) Affymetrix Human Genome U133A 2.0 Array), while GSE30070 was based on the GPL13742 platform (Agilent-015868 Human miRNA Microarray, miRBase release 9.0 feature number version).

Both GSE14209 and GSE30070 were carried out as parallel studies and included 22 and 8 paired pre- and postbiopsy samples, respectively. Endoscopic biopsy samples were collected prior to chemotherapy from metastatic GC patients treated with CF. At the time of disease progression, post-treatment samples were collected from clinical responders. The pre- and post-treatment samples represent 
the chemosensitive and chemoresistant states, respectively.

The study was conducted in accordance with the Declaration of Helsinki (as revised in 2013).

\section{Data pre-processing and identification of DEGs/DEMs}

Using the robust multi-array average method $(11,12)$, the raw data were standardized from a skewed distribution to an approximate normal distribution. It was then normalized with the median standardization method and $\log 2-$ transformed. While a gene symbol may correspond to multiple probes (expression values), the average represented the final expression value for that mRNA or miRNA.

The limma package (13) in $\mathrm{R}$ was used to screen DEGs or DEMs from paired pre- and post-treatment samples. $\mathrm{P}$ value $<0.05$ and $\mid \log \mathrm{FCl}>0.263$ were used as cut-off criteria to identify significant DEGs and DEMs.

\section{Construction of the miRNA-target regulatory network}

The interactions between DEMs and DEM targets were predicted using miRWalk online prediction software (version 3.0, http://mirwalk.umm.uni-heidelberg.de/) (14). Moreover, the predicted targets were validated in 1 or more of the following prediction databases: miRDB release (version 6.0, http://www.mirdb.org/miRDB/download. html/) (15), miRTarBase release (version 7.0, http:// miRTarBase.mbc.nctu.edu.tw/) (16), and TargetScan release (version 7.2, http://www.targetscan.org/) (17). The miRNAmRNA regulatory network based on the correlation analysis of targets and the miRNAs regulating them was visualized using Cytoscape software (version 3.4.0, http://chianti.ucsd. edu/cytoscape-3.4.0/) (18).

\section{Pathway and process enrichment analysis}

To evaluate the DEG and DEM targets at a functional level, the GO functional and KEGG pathway enrichment analyses were performed using clusterProfiler v3.14.0 (https://bioconductor.org/packages/clusterProfiler/). GO functional analysis included 3 functional groups: biological processes (BP), molecular functions (MF), and cellular components (CC). The enriched GO terms and pathways were selected at a $\mathrm{P}$ value $<0.05$.

\section{Construction of the protein-protein interaction network}

An online database, Search Tool for the Retrieval of
Interacting Genes (STRING, version 10.0, http://www. string-db.org/) (19), was used to identify the PPI network among the proteins encoded by the DEGs. A combined score of $>0.4$ was chosen as the cut-off, following which the DEGs in the PPI networks were visualized using Cytoscape software (http://www.cytoscape.org/). Using the TeCytoNCA plug-in (http://apps.cytoscape.org/apps/ cytonca) in Cytoscape software, the important nodes (named hub genes in the PPI network) were obtained based on the topological properties of the network nodes. Furthermore, module analysis of the PPI network was conducted using the molecular complex detection (MCODE) plug-in (http:// apps.cytos cape.org/apps/mcode/). A cut-off level of greater than 5 or 3 was selected as the threshold for statistical significance.

\section{Causal relationships and interactions between proteins}

DisNor (https://disnor.uniroma2.it/) (20) is a new resource designed to infer disease pathways from the disease-related gene identification data. In the present study, DisNor was utilized to depict the key interactions between genes and disease networks. This data was then combined with STRING to illustrate the key gene-protein interaction network. Following direct input of disease names or genes, a regulatory network could be constructed for analysis of the gene relationships when the first neighbor was set as the complexity level.

\section{Survival analysis}

The Kaplan-Meier Plotter (KM-plotter, http://www. kmplot.com/analysis/) is an online database containing gene expression data and survival information of 1,440 clinical GC patients (21). The KM-plotter was utilized to calculate the prognostic value of the hub genes in terms of OS. Patient samples were split at the median expression and only the JetSet best probe set was used. The log-rank P value was calculated and a $\mathrm{P}$ value $<0.05$ was regarded as the cut-off criteria.

\section{Identification of small molecules}

CMap (http://www.broadinstitute.org/cmap/) was used to identify small molecules associated with the identified DEGs. The up-regulated and down-regulated genes in the miRNA-target network were input into the CMap database to identify corresponding small molecules. The 
A

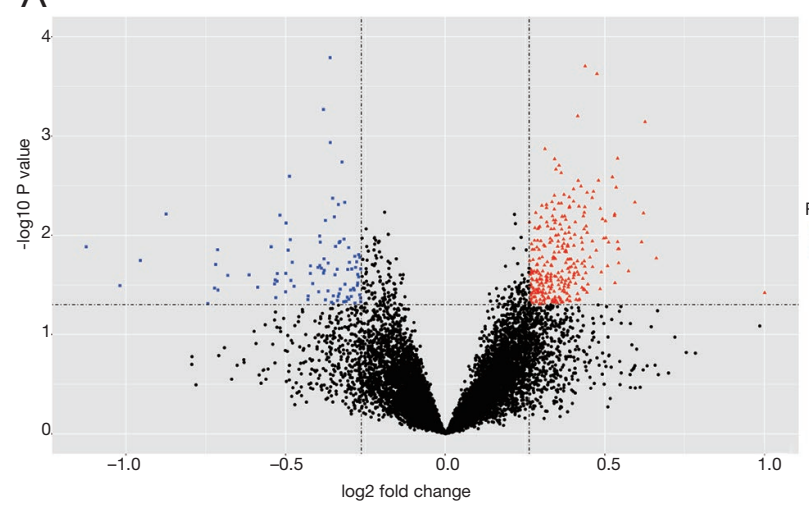

B

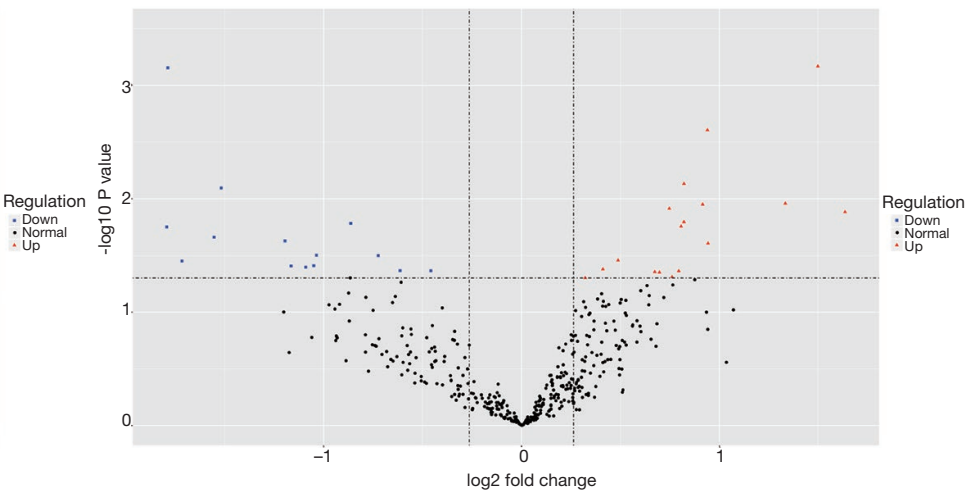

Figure 1 Expression analysis of differentially expressed genes (DEGs) and differentially expressed miRNAs (DEMs) using volcano plots. (A) DEGs in GSE14209 and (B) DEMs in GSE30070. Red indicates significantly up-regulated genes, blue indicates significantly downregulated genes, and black indicates genes with no significant differences in expression.

tool provided an output in the form of connectivity scores ranging from -100 to 100 . A negative connectivity score (closer to -100) demonstrated that the drug was more likely to reverse chemoresistance, and thus might have a therapeutic effect on GC patients with acquired resistance to CF therapy.

\section{Statistical analysis}

Most statistical analyses were performed using $\mathrm{R}$ language and the bioinformatics database online. The identification of DEGs or DEMs was performed based on the criteria of $\mathrm{P}$ value $<0.05$ and $\mid \log \mathrm{FCl}>0.263$ using "limma" package in R. Kaplan-Meier survival plots were generated with survival curves compared by log-rank test. For all analyses, differences were considered statistically significant if $\mathrm{P}$ values were $<0.05$.

\section{Results}

\section{Screening of DEGs and DEMs}

By comparing pre-treatment samples (collected prior to CF therapy) with post-treatment samples collected upon occurrence of acquired resistance (based on objective clinical progression), we identified alterations in gene and miRNA expression levels that might be involved in the acquired resistance to CF chemotherapy in GC patients. The volcano plots in Figure 1 show an overview of DEGs and DEMs in the 2 datasets, with the cut-off criteria of
$\mathrm{P}$ value $<0.05$ and $\mid \log \mathrm{FCl}>0.263$. In summary, a total of 394 DEGs (299 up- and 95 down-regulated), such as the up-regulated mRNA CLDN3 and down-regulated COL11A1, were identified from dataset GSE14209 (Figure 1A). Furthermore, 31 DEMs (17 up- and 14 down-regulated), such as the up-regulated miRNA hsamiR-205-5p and down-regulated hsa-miR-144-3p, were screened from dataset GSE30070 (Figure 1B). The 10 most significant DEGs and DEMs are listed in Tables 1,2.

\section{Analysis of the miRNA-mRNA regulatory network}

Of the 31 DEMs, 29 miRNAs were predicted (along with their 3358 possible targets) by miRWalk2.0 software. There were 57 overlapping DEGs between predicted miRNAtarget genes and DEGs identified in GSE14209. We further mapped the miRNA-mRNA regulatory network, which consisted of 79 nodes, including 57 DEGs and 22 DEMs (Figure 2). Based on rankings according to the degree, the top 10 nodes of miRNAs and mRNAs are listed in Table 3, including hsa-miR-185a-5p (degree =13), hsa-miR-766$3 \mathrm{p}($ degree $=7)$, hsa-miR-198 (degree $=7)$, hsa-miR-145$5 \mathrm{p}($ degree $=7)$, and hsa-miR-629-3p (degree =6). ZBTB44 (degree $=3$ ), which had the highest degree among the DEGs, was co-regulated by hsa-miR-185-5p, hsa-miR548a-3p, and hsa-miR-629-3p. Additionally, TM9SF4 was regulated by both hsa-miR-185a-5p and hsa-miR-145a-5p. Therefore, miRNA and target gene networks can facilitate a better understanding of the mechanisms associated with CF resistance at the molecular level. 
Table 1 The top 10 DEGs identified from two datasets between pre- and post-treatment samples

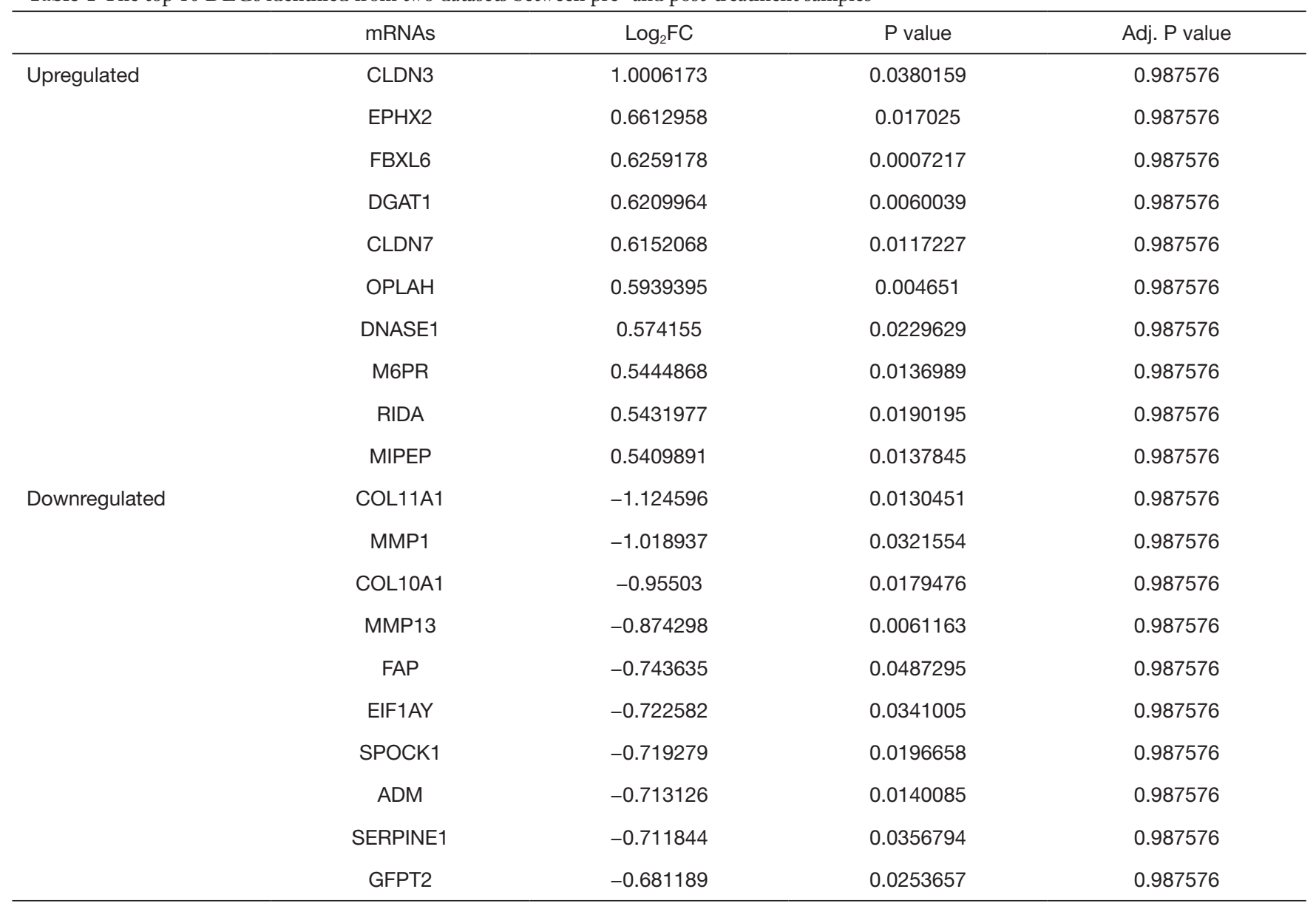

\section{Pathway and process enrichment analyses}

To determine the biological functions of the target DEGs and DEMs, GO functional and KEGG enrichment analyses were performed using clusterProfiler. Upon GO analysis, the DEGs were classified into 3 functional groups: biological processes (BP), molecular function (MF), and cellular components (CC). A total of 23 DEGs were associated with the BP category of ribonucleoprotein complex assembly (GO: 0022618), including AAR2, RPS19, EIF3D, MRPL11, PRPF6, and RC3H1 (Figure 3A). Furthermore, 24 other DEGs were associated with the MF category of catalytic activity acting on RNA (GO: 0140098), including POLRMT, EXOSC4, TRMT112, POLR2L, RRNAD1, and WARS2 (Figure 3B). Additionally, 44 DEGs were associated with the CC category of mitochondrial matrix (GO: 0005759), including TXN2, TUFM, POLRMT, ACP6, PAM16, and IDH3B (Figure 3C). Moreover, 14 DEGs were found to be enriched in the thermogenesis KEGG pathway
(Figure 3D). DEM targets were predominantly involved in single-stranded RNA binding (GO: 0003727), which consisted of RBMS3, ILF3, and PPIE in the MF category (Figure 4A). Some DEMs participated in the endoplasmic reticulum lumen (GO: 0005788) in the CC category, including 6 targets such as CLN6, CST3, ERP29, GANAB, APLP2, and COL10A1 (Figure 4B). However, no DEMs were found to be significantly enriched in any of the GO terms of BP or the KEGG pathway.

\section{PPI network construction}

Using the STRING database and Cytoscape software, a PPI network was built based on selection criteria. The nodes were targets of DEMs and the network comprised a set of 105 pairs and 53 nodes, including 31 up-regulated and 22 down-regulated DEGs (Figure $5 A$ ). The degree of every node was calculated and filtered upon evaluating the 
Table 2 The top 10 DEMs identified from two datasets between pre- and post-treatment samples

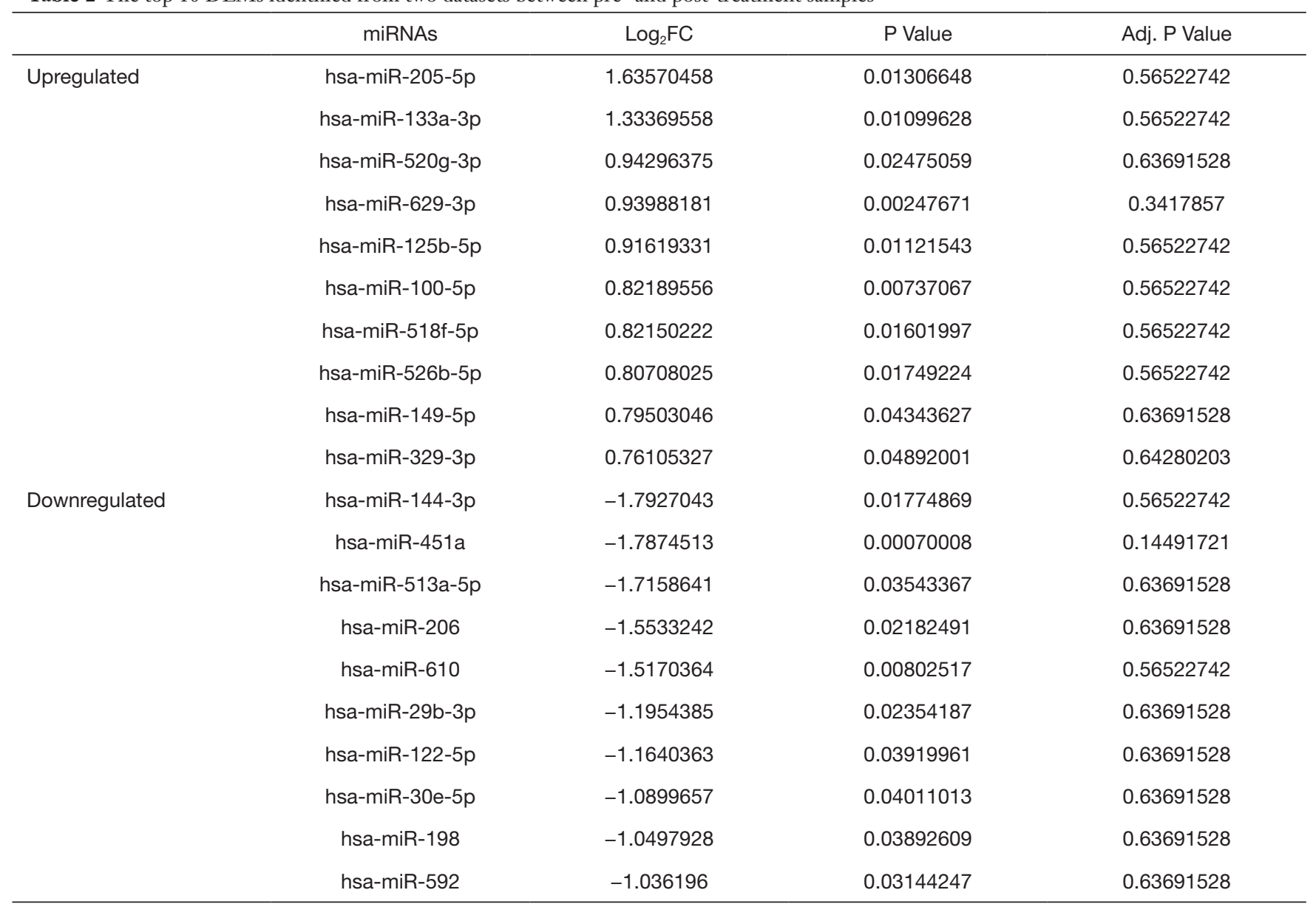

topological properties of the PPI network. The top 10 genes, including HDAC5 (degree =12), DDX17 (degree =10), ILF3 (degree $=10)$, and SDHC (degree $=10)$, were then selected as hub genes (Table 4). All 4 hub genes were up-regulated in the state of acquired resistance, thus indicating that they might contribute to CF chemoresistance. Additionally, the top 3 most significant modules were screened by the MCODE plug-in. Module 1 contained 5 DEGs (PPIE, HDAC5, SF1, DHX35, and DDX17; Figure 5B), module 2 included 3 nodes (ILF3, LSM12, and COMMD9; Figure 5C), and module 3 consisted of 3 DEGs (DPAGT1, TM9SF4, and GANAB; Figure 5D).

\section{Causal relationships and interactions with proteins}

DisNor identified the first neighbors of the key genes, among which PTPN6, CBL, PTPN1, AKT1, and PRKCA were found to up-regulate or down-regulate at least 4 key genes involved in the cellular membrane and cytoplasm. For example, PRKCA was upstream to and inhibited the genes PTPN6 and CBL, along with suppressing INSR. Furthermore, PTPN6, CBL, PTPN1, AKT1, and PRKCA interacted with each other (Figure 6).

\section{Survival analysis of bub genes}

Next, we evaluated the prognostic effects of the 4 hub genes on GC patients. The Kaplan-Meier curves indicated that GC patients with high expression levels of the genes HDAC5, DDX17, and ILF3 had worse OS $(\mathrm{P}<0.05)$, while GC patients with high expression levels of the gene SDHC had better OS $(\mathrm{P}<0.05$; Figure 7$)$.

\section{Identification of related active small molecules}

For the purpose of screening out candidate drugs, up- 


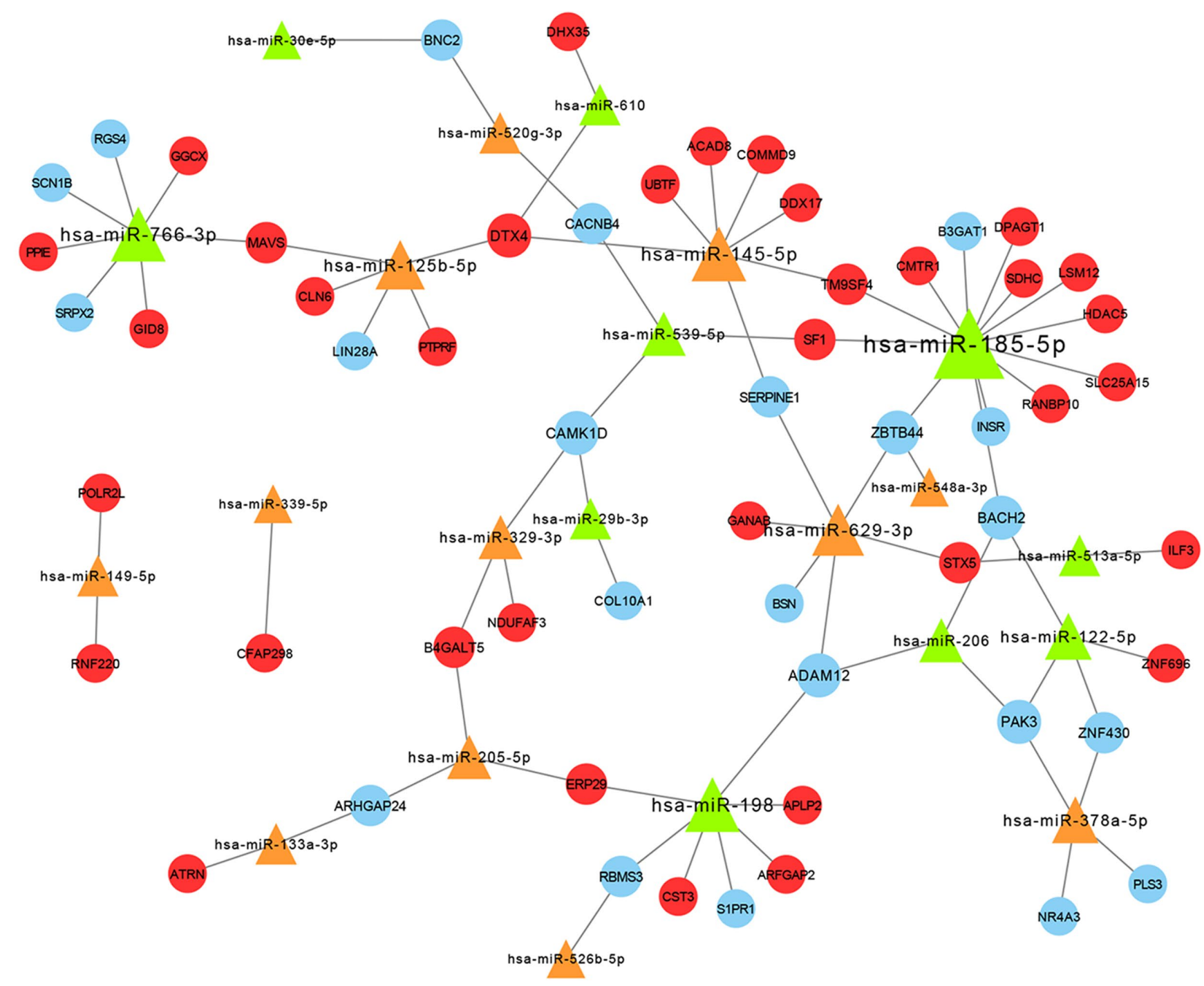

Figure 2 Analysis of the miRNA-mRNA regulatory network. Representations are as follows: blue circular nodes, down-regulated mRNAs; red circular nodes, up-regulated mRNAs; green triangles, down-regulated miRNAs; orange triangles, up-regulated miRNAs.

regulated and down-regulated gene groups between the pretreatment and post-treatment samples were queried in the CMap database. The top 10 small molecules corresponding to GC-related alterations in gene expression and their predicted targets are listed in Table 5. Three types of drugs, including mitochondrial DNA polymerase inhibitors, histone deacetylase inhibitors (HDACIs), and T-type calcium channel blockers, were identified. Among these small molecules, menadione (score $=-93.23$ ), belinostat (score $=-82.95$ ), and NCH-51 (score $=-76.62$ ) showed the highest negative correlations and the potential to reverse chemoresistance of GC.

\section{Discussion}

The last few years have seen a surge in the applications of bioinformatics analysis in clinical studies. This can be credited to the rapid developments in microarray and high-throughput technologies. The GEO database, a public high-throughput gene molecular abundance database, provides advanced tools to evaluate key genes and molecular pathways involved in tumorigenesis. The high chemoresistance and cytotoxicity of CF therapy limits its use in advanced GC patients. Partial or complete responders to $\mathrm{CF}$ therapy often develop drug resistance in a short time, leading to therapeutic failure. A 72 - 
Table 3 Top 10 nodes of miRNAs and mRNAs in the miRNAmRNA regulatory network, based on the degree

\begin{tabular}{lcc}
\hline miRNA & Description & Degree \\
\hline hsa-miR-185-5p & Down & 13 \\
hsa-miR-766-3p & Down & 7 \\
hsa-miR-198 & Down & 7 \\
hsa-miR-145-5p & Up & 7 \\
hsa-miR-629-3p & Up & 6 \\
hsa-miR-125b-5p & Up & 5 \\
hsa-miR-378a-5p & Up & 4 \\
hsa-miR-122-5p & Down & 4 \\
ZBTB44 & Down & 3 \\
hsa-miR-539-5p & Down & 3 \\
\hline
\end{tabular}

gene acquired resistance signature was identified as an independent predictor for time to progression and survival of CF treated patients (22). Studies have identified some molecular mechanisms for the same, including alterations in cellular platinum accumulation, increased detoxification systems, increased DNA repair, decreased apoptosis, and autophagy (23). However, the underlying mechanisms remain to be elucidated. In this study, thanks to the technical feasibility, it is possible to obtain endoscopic biopsy samples from the same patients after the occurrence of acquired resistance. Using the GEO database, we screened a total of 394 DEGs from GSE14209 and 31 DEMs from GSE30070 by comparing pre- and post-treatment samples. We then used miRWalk to predict the target genes of the DEMs, which led to the screening of 3,358 potential target genes.
A

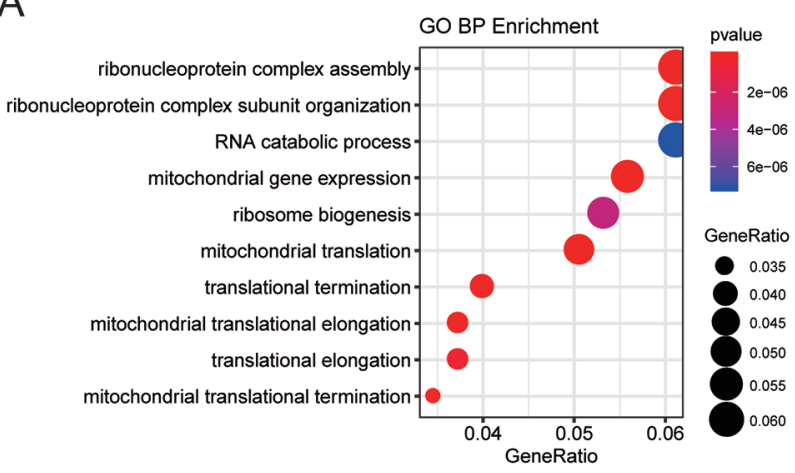

C

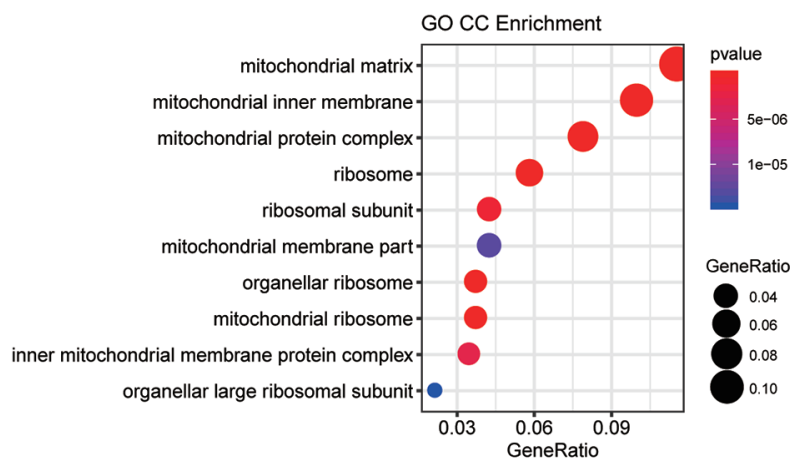

B

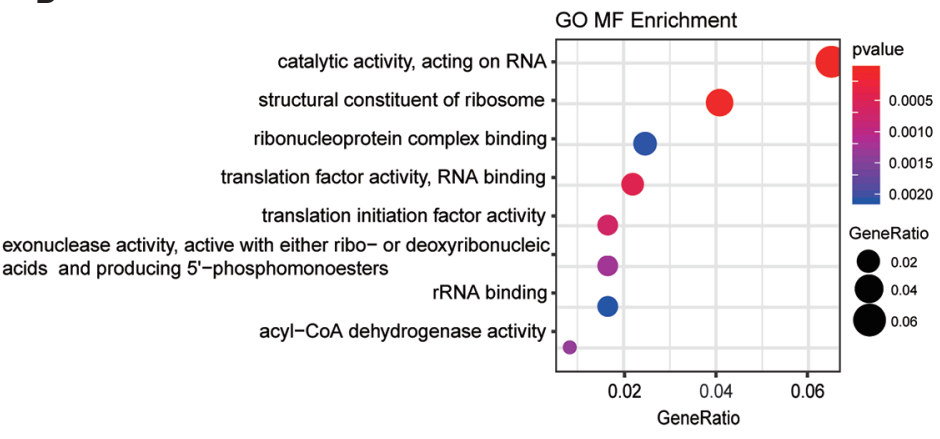

D

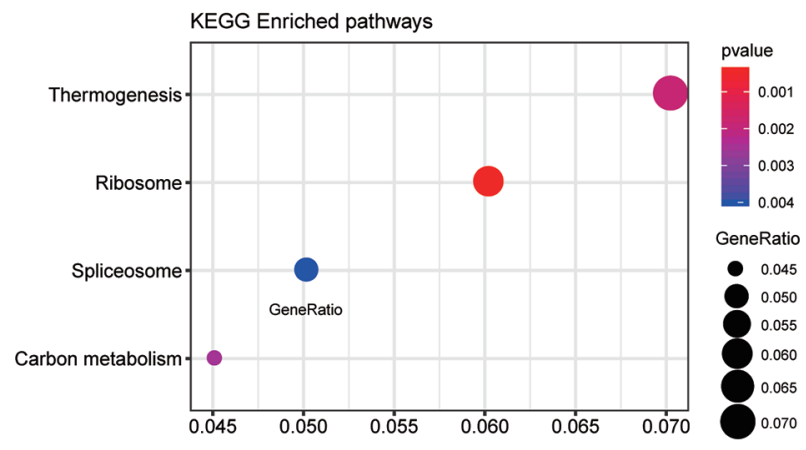

Figure 3 Functional and signaling pathway analyses of DEGs. (A) Biological processes, (B) molecular function, (C) cellular components, and (D) KEGG pathway. DEG, differentially expressed gene; KEGG, GKyoto Encyclopedia of Genes and Genomes. 
A

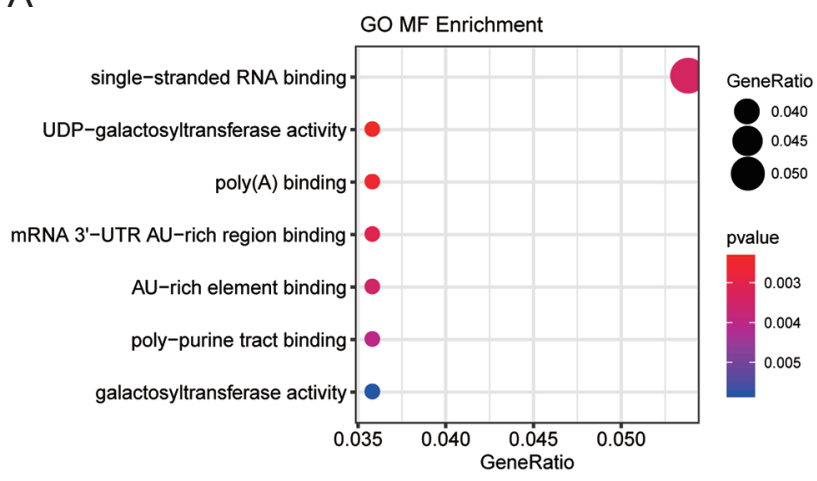

B

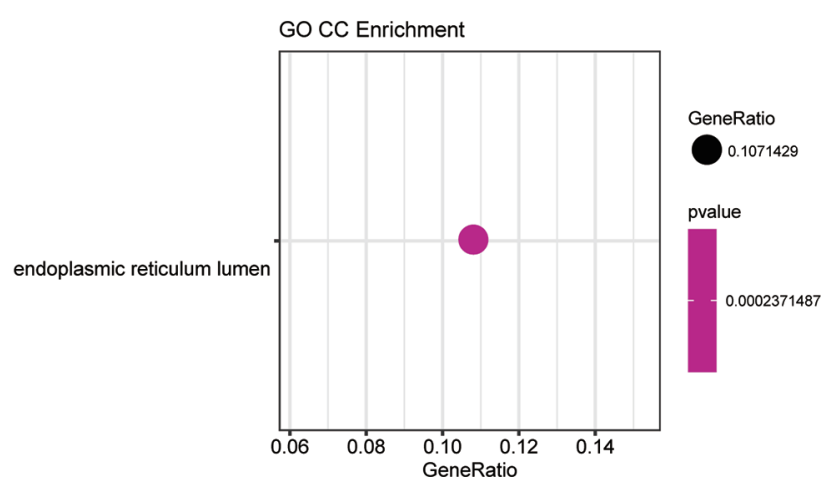

Figure 4 Functional and signaling pathway analyses of differentially expressed miRNAs (DEMs). (A) Molecular function and (B) cellular components.

A

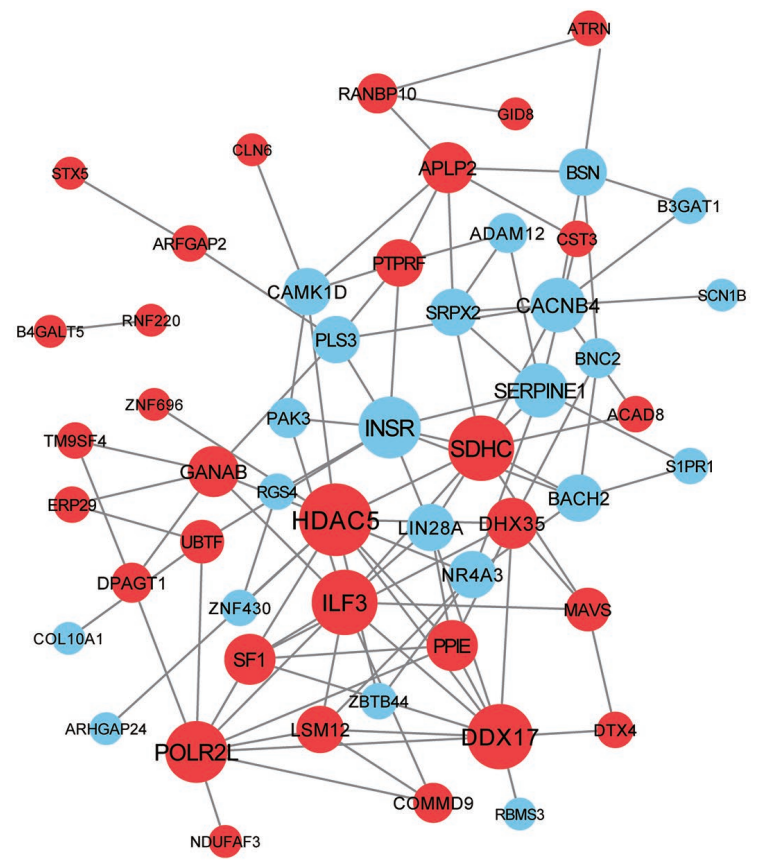

B

C
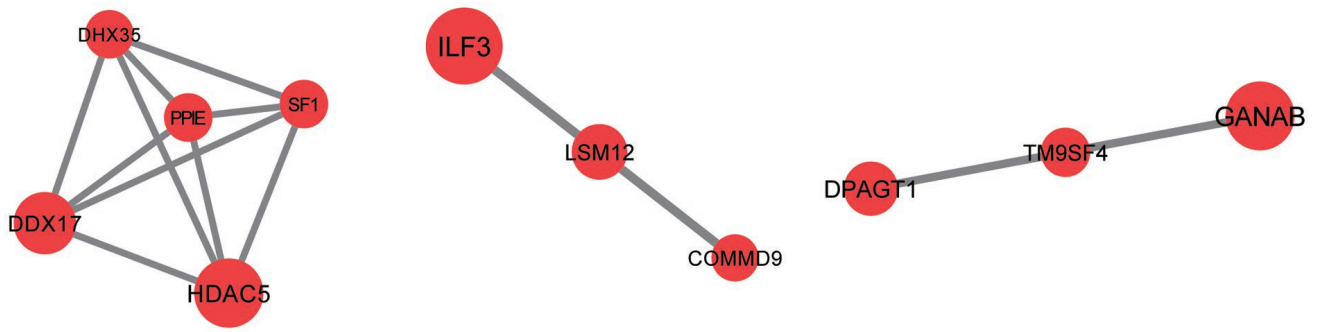

Figure 5 The protein-protein interaction (PPI) network among overlapping DEGs. (A) PPI network of proteins encoded by DEGs; (B) Module 1 contained five nodes and nine edges; (C) Module 2 contained three nodes and two edges; (D) Module 3 contained three nodes and two edges. DEG, differentially expressed gene. 
Of these potential genes, 57 were then identified as DEGs and carried forward for further analysis. In the case of DEGs, GO and KEGG showed that these were most significantly enriched in the processes of ribonucleoprotein complex assembly, catalytic activity acting on RNA, mitochondrial matrix, and thermogenesis.

The miRNA-mRNA regulatory networks predicted that hsa-miR-185-5p, hsa-miR-145-5p, and TM9SF4 may

Table 4 Top 10 genes in the protein-protein interaction (PPI) network

\begin{tabular}{lcc}
\hline Node & Description & Degree \\
\hline HDAC5 & Up & 12 \\
DDX17 & Up & 10 \\
ILF3 & Up & 10 \\
SDHC & Up & 10 \\
INSR & Down & 9 \\
POLR2L & Up & 9 \\
CACNB4 & Down & 7 \\
SERPINE1 & Down & 7 \\
APLP2 & Up & 6 \\
SF1 & Up & 6 \\
\hline
\end{tabular}

contribute to the development of drug resistance in GC patients. GO and KEGG showed that the DEMs were most significantly enriched in the processes of single-stranded RNA binding and endoplasmic reticulum lumen. Various studies have shown that hsa-mir-185-5p acts as a tumorsuppressor and is down-regulated in GC tissues $(24,25)$, and hsa-miR-185 has been identified as an independent prognostic factor that suppresses tumor metastasis in GC (26). Furthermore, hsa-mir-185 has been shown to be involved in the regulation of chemotherapeutic sensitivity in GC by targeting the apoptosis repressor with caspase recruitment domain (27). Interestingly, down-regulated $\mathrm{miR}-145-5 \mathrm{p}$ is associated with poor prognosis in GC (28). Overexpression of miR-145-5p has been shown to suppress the migration and invasion of prostate cancer cells, in addition to reducing the chemoresistance to docetaxel (29). According to the miRNA-mRNA regulatory network in the present study, TM9SF4 was a target of hsa-miR-185$5 \mathrm{p}$ and hsa-miR-145-5p. A previous study reported that knockdown of TM9SF4 alleviated endoplasmic reticulum stress and protected chemoresistant breast cancer cells from cell death (30). TM9SF4 interacts with V-ATPase in colon cancer cells, thus playing an important role in the resistance to 5 -fluorouracil (31).

The PPI network suggested that HDAC5, DDX17,

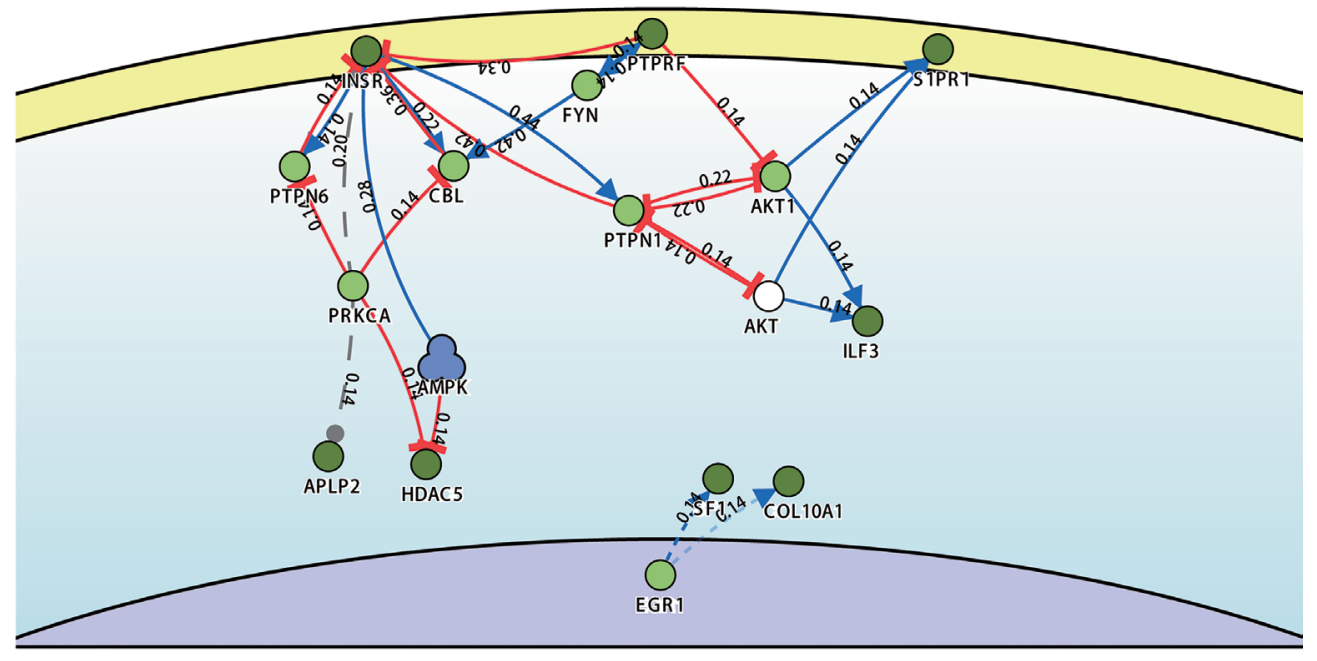

Figure 6 Analysis of causal interaction between key genes using DisNor. The blue line with arrowhead suggests activation, red line with T arrow suggests inhibition, and grey dashed line means unknown effect. The distance of each path was calculated from the reliability score, $r$. 
A

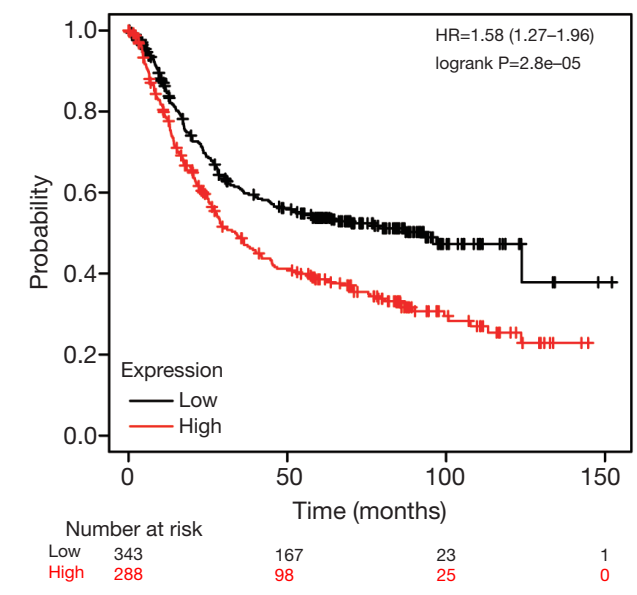

C

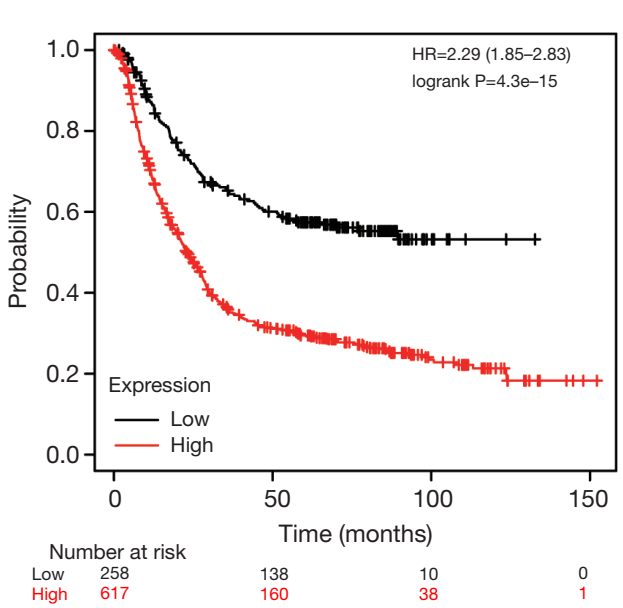

B

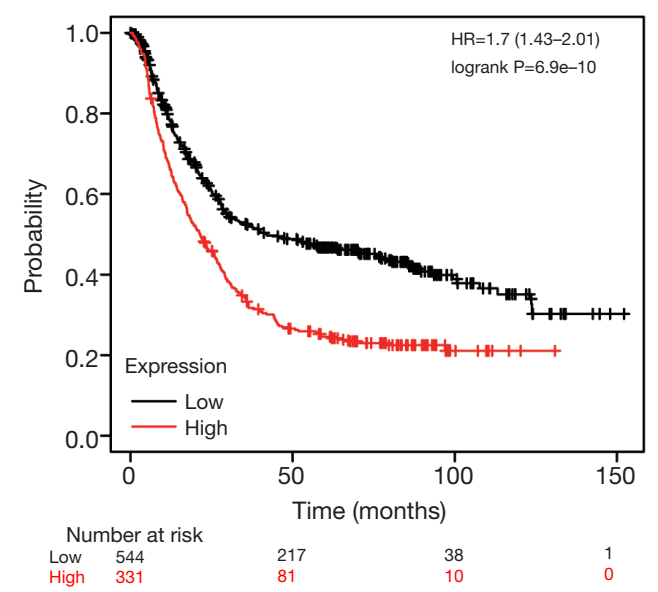

$\mathrm{SDHC}$

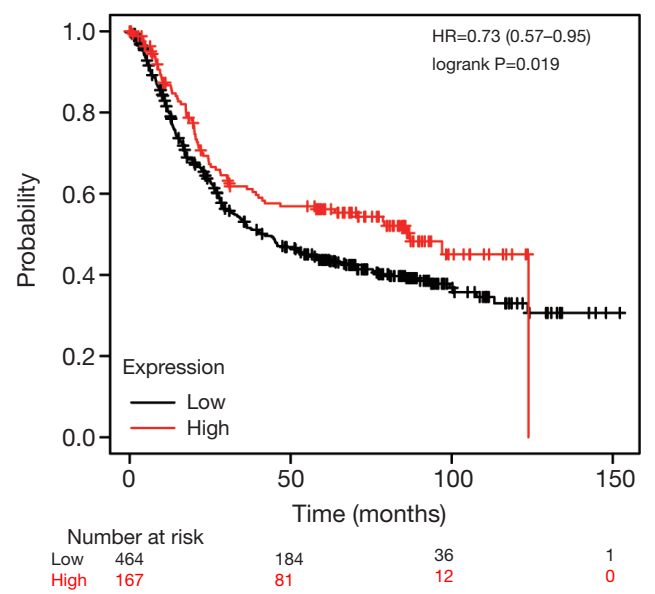

Figure 7 Prognostic values of hub genes in GC patients. OS curves were plotted to evaluate the prognostic value of the mRNA expression levels of the hub genes. The log-rank test was carried out using the relevant results. (A) HDAC5 (229408_at), (B) DDX17 (208718_at), (C) ILF3 (217804_s_at), and (D) SDHC (238056_at).

ILF3, and SDHC might be critical for the development of chemoresistance in GC. Aberrant activation of HDACs in cancer cells results in the modulation of cell proliferation, migration, differentiation, and metastasis $(31,32)$. For example, overexpression of HDACs is an independent prognostic marker in GC treatment (33). ILF3 is related to multiple cancers, such as hepatocellular carcinoma, non-small cell lung carcinoma, breast cancer, and ovarian cancer (34-38). ILF3 may function as a transcriptional coactivator, and regulates tumor proliferation and metastasis. ILF3 results in the deterioration of GC by promoting the proliferation of GC cells, and the detection of this protein may be useful in the prognostic prediction of GC patients (39). However, its role in the drug resistance of
GC patients remains unclear. DDX17 is an oncogene in various cancers, and is associated with acquired gefitinib resistance in non-small cell lung cancer (40-43). SDHC is 1 of the 4 subunits of the succinate dehydrogenase complex in the mitochondrial electron transport chain. Epigenetic modification of SDHC is associated with cancer progression $(44,45)$. Our survival analysis revealed that high expression of hub genes, including HDAC5, DDX17, and ILF3, was significantly correlated with worse OS of GC patients, indicating that these hub genes might play important roles in the progression of GC. However, the complex mechanisms of these hub genes remain to be investigated.

CMap was developed to identify bioactive small molecules that induced similar or opposite gene-expression profiles in 
Table 5 Top candidate drugs, post analysis by CMap

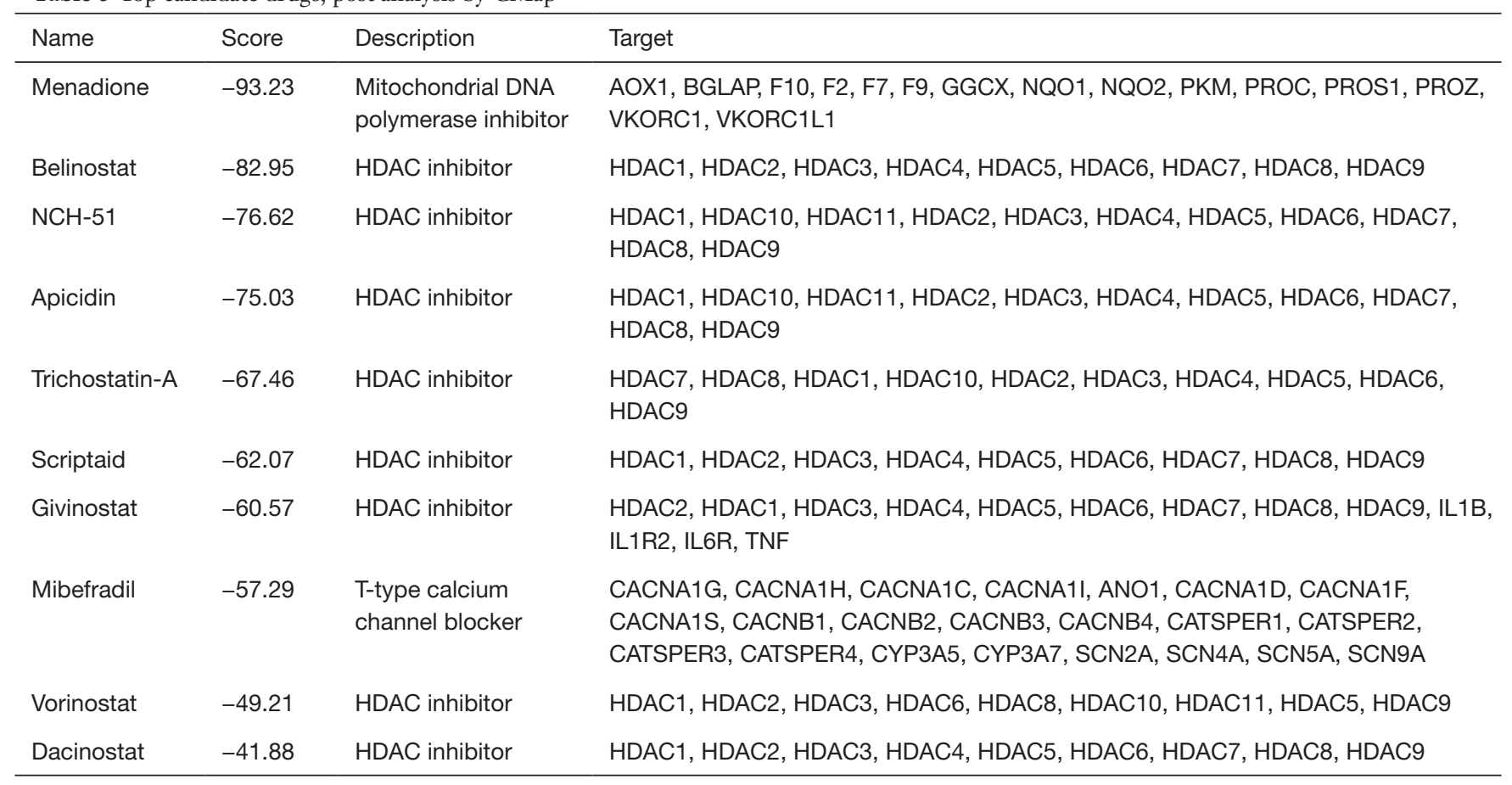

different diseases. Small molecules with a strong negative connectivity score are identified as ones with a therapeutic effect on the disease. CMap is an interesting tool to discover new functions and applications for available drugs, and allows for quicker testing of the drugs in clinical trials, compared to newly developed drugs $(46,47)$. In this study, a panel of small molecule drugs was screened by comparing the DEGs with the CMap database. These drugs showed promise in reversing the alterations in gene expression of GC patients displaying resistance to chemotherapy.

Menadione, also known as vitamin $\mathrm{K} 3$, is an essential nutrient for blood clotting, calcium regulation, and bone metabolism. Menadione has been reported to exhibit anticancer activity in vitro, primarily by generating reactive oxygen species, which lead to DNA damage and cell death (48-50). Previous studies have demonstrated that in GC, menadione induces cell cycle arrest and apoptosis, and inhibits tumor development and progression in vivo (51-53). Several clinical trials have evaluated the synergistic cytotoxicity of menadione with other anticancer drugs. A clinical phase I/IIa study involving vitamin $\mathrm{C}$ and vitamin $\mathrm{K} 3$ showed the safety and efficacy of this combination in delaying biochemical progression in end-stage prostate cancer patients who had failed standard therapy (54). A few novel menadione hybrids have also been designed and synthesized, and have demonstrated significant anticancer activities against cancer cell lines (55). Therefore, menadione may be an attractive therapeutic target in GC, especially for patients in more advanced stages (56).

T-type $\mathrm{Ca}^{2+}$ channels, which may regulate a variety of $\mathrm{Ca}^{2+}$-dependent cellular processes, such as cell proliferation, migration, survival, and differentiation, are overexpressed in various cancers. Therefore, T-type $\mathrm{Ca}^{2+}$ channels have the potential to be promising therapeutic targets for cancer interventions.

HDACs are capable of deacetylating the lysine residues of histones and some non-histone proteins. Aberrant expression and activity of HDACs are associated with the development of various cancers. Thus, suppression of HDAC activity is now gaining attention as a promising anticancer therapy. So far, 4 HDACIs have already been approved for cancer treatment by the Food and Drug Administration, including: (I) vorinostat (SAHA) for cutaneous T-cell lymphoma (57); (II) belinostat (PXD101) for peripheral T-cell lymphomas (58); (III) romidepsin (FK228) for both cutaneous and peripheral T-cell lymphomas (59); and (IV) panobinostat for myeloma (LBH-589) (60). Although HDACIs have been highly successful in cases of hematological malignancies, singleagent epigenetic therapy appeared to be less effective 
against solid tumors. The non-selective inhibition of HDACIs against pan-HDAC isoforms results in severe adverse effects and toxicities (61). Therefore, there is an urgent need to develop new isoform-selective HDACIs. Additionally, the use of HDACIs in combination with chemotherapy or immunotherapy is expected to improve the effectiveness of conventional drugs and survival rates. For instance, vorinostat combined with capecitabine and cisplatin is a feasible first-line chemotherapy for treating patients with advanced GC (62). Vorinostat, in combination with carboplatin and paclitaxel, has been shown to have enhanced anticancer effects in advanced non-small cell lung carcinoma patients, leading to improved survival (63). These findings indicate that epigenetic drugs hold promise for improving the benefits of conventional therapies, though further studies are needed to confirm this.

Although the problem of drug resistance is difficult to solve, some possible solutions have been suggested, including earlier detection of tumors, deeper therapeutic responses, adaptive monitoring during therapy and mapping cancer dependencies (64). Considering the limitations of single biological treatment, combination therapy is the direction of future development that may achieve good results.

\section{Conclusions}

Using integrated bioinformatics analysis, the present study expands our knowledge of the molecular signatures for acquired resistance to CF therapy in GC. We also identified hub genes in the PPI network, including HDAC5, DDX17, and ILF3, which might play important roles in the development of acquired chemoresistance. Additionally, this study also led to the identification of some active small molecules, such as HDACIs, menadione, and mibefradil, which could potentially be utilized for GC treatment. However, since our results are based on bioinformatics analysis, these serve as predictions and need to be further validated using in vitro and in vivo experiments.

\section{Acknowledgments}

Funding: This work was supported by the Scientific Research Projects of Wuxi Health Commission (Q201819).

\section{Footnote}

Reporting Checklist: The authors have completed the Materials Design Analysis Reporting (MDAR) reporting checklist. Available at http://dx.doi.org/10.21037/jgo-21-81

Conflicts of Interest: All authors have completed the ICMJE uniform disclosure form (available at http://dx.doi. org/10.21037/jgo-21-81). The authors have no conflicts of interest to declare.

Ethical Statement: The authors are accountable for all aspects of the work in ensuring that questions related to the accuracy or integrity of any part of the work are appropriately investigated and resolved. The study was conducted in accordance with the Declaration of Helsinki (as revised in 2013).

Open Access Statement: This is an Open Access article distributed in accordance with the Creative Commons Attribution-NonCommercial-NoDerivs 4.0 International License (CC BY-NC-ND 4.0), which permits the noncommercial replication and distribution of the article with the strict proviso that no changes or edits are made and the original work is properly cited (including links to both the formal publication through the relevant DOI and the license). See: https://creativecommons.org/licenses/by-nc-nd/4.0/.

\section{References}

1. Bray F, Ferlay J, Soerjomataram I, et al. Global cancer statistics 2018: GLOBOCAN estimates of incidence and mortality worldwide for 36 cancers in 185 countries. CA Cancer J Clin 2018;68:394-424.

2. Sitarz R, Skierucha M, Mielko J, et al. Gastric cancer: epidemiology, prevention, classification, and treatment. Cancer Manag Res 2018;10:239-48.

3. Zong $\mathrm{L}$, Abe $\mathrm{M}$, Seto $\mathrm{Y}$, et al. The challenge of screening for early gastric cancer in China. Lancet 2016;388:2606.

4. Li B, Chen L, Luo HL, et al. Docetaxel, cisplatin, and 5 -fluorouracil compared with epirubicin, cisplatin, and 5-fluorouracil regimen for advanced gastric cancer: A systematic review and meta-analysis. World J Clin Cases 2019;7:600-15.

5. Reuter JA, Spacek DV, Snyder MP. High-throughput sequencing technologies. Mol Cell 2015;58:586-97.

6. Tao Z, Shi A, Li R, et al. Microarray bioinformatics in cancer- a review J Buon 2017;22:838-43.

7. Lamb J. The Connectivity Map: a new tool for biomedical research. Nat Rev Cancer 2007;7:54-60.

8. Lamb J, Crawford ED, Peck D, et al. The Connectivity Map: using gene-expression signatures to connect small 
molecules, genes, and disease. Science 2006;313:1929-35.

9. Chen J, Wang Z, Shen X, et al. Identification of novel biomarkers and small molecule drugs in human colorectal cancer by microarray and bioinformatics analysis. Mol Genet Genomic Med 2019;7:e00713.

10. Wu Q, Zhang B, Sun Y, et al. Identification of novel biomarkers and candidate small molecule drugs in nonsmall-cell lung cancer by integrated microarray analysis. Onco Targets Ther 2019;12:3545-63.

11. Bolstad BM, Irizarry RA, Astrand M, et al. A comparison of normalization methods for high density oligonucleotide array data based on variance and bias. Bioinformatics 2003;19:185-93.

12. Irizarry RA, Hobbs B, Collin F, et al. Exploration, normalization, and summaries of high density oligonucleotide array probe level data. Biostatistics 2003;4:249-64.

13. Ritchie ME, Phipson B, Wu D, et al. limma powers differential expression analyses for RNA-sequencing and microarray studies. Nucleic Acids Res 2015;43:e47.

14. Sticht C, De La Torre C, Parveen A, et al. miRWalk: An online resource for prediction of microRNA binding sites. PLoS One 2018;13:e0206239.

15. Wong N, Wang X. miRDB: an online resource for microRNA target prediction and functional annotations. Nucleic Acids Res 2015;43:D146-52.

16. Chou CH, Shrestha S, Yang CD, et al. miRTarBase update 2018: a resource for experimentally validated microRNAtarget interactions. Nucleic Acids Res 2018;46:D296-302.

17. Agarwal V, Bell GW, Nam JW, et al. Predicting effective microRNA target sites in mammalian mRNAs. Elife 2015;4:e05005.

18. Shannon P, Markiel A, Ozier O, et al. Cytoscape: a software environment for integrated models of biomolecular interaction networks. Genome Res 2003;13:2498-504.

19. Szklarczyk D, Gable AL, Lyon D, et al. STRING v11: protein-protein association networks with increased coverage, supporting functional discovery in genomewide experimental datasets. Nucleic Acids Res 2019;47:D607-13.

20. Lo Surdo P, Calderone A, Iannuccelli M, et al. DISNOR: a disease network open resource. Nucleic Acids Res 2018;46:D527-34.

21. Nagy Á, Lánczky A, Menyhárt O, et al. Validation of miRNA prognostic power in hepatocellular carcinoma using expression data of independent datasets. Sci Rep 2018;8:9227.
22. Kim HK, Choi IJ, Kim CG, et al. A gene expression signature of acquired chemoresistance to cisplatin and fluorouracil combination chemotherapy in gastric cancer patients. PLoS One 2011;6:e16694.

23. Zhou J, Kang Y, Chen L, et al. The Drug-Resistance Mechanisms of Five Platinum-Based Antitumor Agents. Front Pharmacol 2020;11:343.

24. Yuan C, Zhang Y, Tu W, et al. Integrated miRNA profiling and bioinformatics analyses reveal upregulated miRNAs in gastric cancer. Oncol Lett 2019;18:1979-88.

25. Bibi F, Naseer MI, Alvi SA, et al. microRNA analysis of gastric cancer patients from Saudi Arabian population. BMC Genomics 2016;17:751.

26. Tan Z, Jiang H, Wu Y, et al. miR-185 is an independent prognosis factor and suppresses tumor metastasis in gastric cancer. Mol Cell Biochem 2014;386:223-31.

27. Li Q, Wang JX, He YQ, et al. MicroRNA-185 regulates chemotherapeutic sensitivity in gastric cancer by targeting apoptosis repressor with caspase recruitment domain. Cell Death Dis 2014;5:e1197.

28. Zhang Y, Wen X, Hu XL, et al. Downregulation of miR$145-5$ p correlates with poor prognosis in gastric cancer. Eur Rev Med Pharmacol Sci 2016;20:3026-30.

29. Xue D, Lu H, Xu HY, et al. Long noncoding RNA MALAT1 enhances the docetaxel resistance of prostate cancer cells via miR-145-5p-mediated regulation of AKAP12. J Cell Mol Med 2018;22:3223-37.

30. Zhu Y, Xie M, Meng Z, et al. Knockdown of TM9SF4 boosts ER stress to trigger cell death of chemoresistant breast cancer cells. Oncogene 2019;38:5778-91.

31. Lozupone F, Borghi M, Marzoli F, et al. TM9SF4 is a novel V-ATPase-interacting protein that modulates tumor $\mathrm{pH}$ alterations associated with drug resistance and invasiveness of colon cancer cells. Oncogene 2015;34:5163-74.

32. Yoo CB, Jones PA. Epigenetic therapy of cancer: past, present and future. Nat Rev Drug Discov 2006;5:37-50.

33. Weichert W, Röske A, Gekeler V, et al. Association of patterns of class I histone deacetylase expression with patient prognosis in gastric cancer: a retrospective analysis. Lancet Oncol 2008;9:139-48.

34. Guo NL, Wan YW, Tosun K, et al. Confirmation of gene expression-based prediction of survival in non-small cell lung cancer. Clin Cancer Res 2008;14:8213-20.

35. Guo Y, Fu P, Zhu H, et al. Correlations among ERCC1, XPB, UBE2I, EGF, TAL2 and ILF3 revealed by gene signatures of histological subtypes of patients with epithelial ovarian cancer. Oncol Rep 2012;27:286-92. 
36. Hu Q, Lu YY, Noh H, et al. Interleukin enhancer-binding factor 3 promotes breast tumor progression by regulating sustained urokinase-type plasminogen activator expression. Oncogene 2013;32:3933-43.

37. Jiang W, Huang H, Ding L, et al. Regulation of cell cycle of hepatocellular carcinoma by NF90 through modulation of cyclin E1 mRNA stability. Oncogene 2015;34:4460-70.

38. Zhang $\mathrm{Y}$, Yang $\mathrm{C}$, Zhang $\mathrm{M}$, et al. Interleukin enhancerbinding factor 3 and $\mathrm{HOXC} 8$ co-activate cadherin 11 transcription to promote breast cancer cells proliferation and migration. Oncotarget 2017;8:107477-91.

39. Liu Y, Li Y, Zhao Y, et al. ILF3 promotes gastric cancer proliferation and may be used as a prognostic marker. Mol Med Rep 2019;20:125-34.

40. Kao SH, Cheng WC, Wang YT, et al. Regulation of miRNA Biogenesis and Histone Modification by K63Polyubiquitinated DDX17 Controls Cancer Stem-like Features. Cancer Res 2019;79:2549-63.

41. Xue Y, Jia X, Li C, et al. DDX17 promotes hepatocellular carcinoma progression via inhibiting Klf4 transcriptional activity. Cell Death Dis 2019;10:814.

42. Alqahtani H, Gopal K, Gupta N, et al. DDX17 (P72), a Sox 2 binding partner, promotes stem-like features conferred by Sox 2 in a small cell population in estrogen receptor-positive breast cancer. Cell Signal 2016;28:42-50.

43. Li K, Mo C, Gong D, et al. DDX17 nucleocytoplasmic shuttling promotes acquired gefitinib resistance in nonsmall cell lung cancer cells via activation of $\beta$-catenin. Cancer Lett 2017;400:194-202.

44. Casey RT, Ten Hoopen R, Ochoa E, et al. SDHC epimutation testing in gastrointestinal stromal tumours and related tumours in clinical practice. Sci Rep 2019;9:10244.

45. Li J, Liang N, Long X, et al. SDHC-related deficiency of SDH complex activity promotes growth and metastasis of hepatocellular carcinoma via ROS/NFאB signaling. Cancer Lett 2019;461:44-55.

46. Yu L, Yao S, Gao L, et al. Conserved Disease Modules Extracted From Multilayer Heterogeneous Disease and Gene Networks for Understanding Disease Mechanisms and Predicting Disease Treatments. Front Genet 2019;9:745.

47. Yu L, Su R, Wang B, et al. Prediction of Novel Drugs for Hepatocellular Carcinoma Based on Multi-Source Random Walk. IEEE/ACM Trans Comput Biol Bioinform 2017;14:966-77.

48. Marchionatti AM, Picotto G, Narvaez CJ, et al. Antiproliferative action of menadione and 1,25(OH)2D3 on breast cancer cells. J Steroid Biochem Mol Biol
2009;113:227-32.

49. Oztopcu-Vatan P, Sayitoglu M, Gunindi M, et al. Cytotoxic and apoptotic effects of menadione on rat hepatocellular carcinoma cells. Cytotechnology 2015;67:1003-9.

50. Ren X, Santhosh SM, Coppo L, et al. The combination of ascorbate and menadione causes cancer cell death by oxidative stress and replicative stress. Free Radic Biol Med 2019;134:350-8.

51. Bona AB, Calcagno DQ, Ribeiro HF, et al. Menadione reduces CDC25B expression and promotes tumor shrinkage in gastric cancer. Therapeutic Advances in Gastroenterology 2020;13:1-13.

52. Lee MH, Cho Y, Kim DH, et al. Menadione induces G2/ $M$ arrest in gastric cancer cells by down-regulation of CDC25C and proteasome mediated degradation of CDK1 and cyclin B1. Am J Transl Res 2016;8:5246-55.

53. Lee MH, Yang JY, Cho Y, et al. Menadione induces apoptosis in a gastric cancer cell line mediated by downregulation of $\mathrm{X}$-linked inhibitor of apoptosis. International Journal of Clinical and Experimental Medicine 2016;9:2437-43.

54. Tareen B, Summers JL, Jamison JM, et al. A 12 week, open label, phase I/IIa study using apatone for the treatment of prostate cancer patients who have failed standard therapy. Int J Med Sci 2008;5:62-7.

55. Prasad CV, Nayak VL, Ramakrishna S, Mallavadhani UV. Novel menadione hybrids: Synthesis, anticancer activity, and cell-based studies. Chem Biol Drug Des 2018;91:220-33.

56. Oh SJ, Han HK, Kang KW, et al. Menadione serves as a substrate for P-glycoprotein: implication in chemosensitizing activity. Arch Pharm Res 2013;36:509-16.

57. Mann BS, Johnson JR, Cohen MH, Justice R, Pazdur R. FDA approval summary: vorinostat for treatment of advanced primary cutaneous T-cell lymphoma. Oncologist 2007;12:1247-52.

58. McDermott J, Jimeno A. Belinostat for the treatment of peripheral T-cell lymphomas. Drugs Today (Barc) 2014;50:337-45.

59. Bertino EM, Otterson GA. Romidepsin: a novel histone deacetylase inhibitor for cancer. Expert Opin Investig Drugs 2011;20:1151-8.

60. Richardson PG, Laubach JP, Lonial S, et al. Panobinostat: a novel pan-deacetylase inhibitor for the treatment of relapsed or relapsed and refractory multiple myeloma. Expert Rev Anticancer Ther 2015;15:737-48.

61. Guha M. HDAC inhibitors still need a home run, despite 
recent approval. Nat Rev Drug Discov 2015;14:225-6.

62. Yoo C, Ryu MH, Na YS, et al. Vorinostat in combination with capecitabine plus cisplatin as a first-line chemotherapy for patients with metastatic or unresectable gastric cancer: phase II study and biomarker analysis. Br J Cancer 2016;114:1185-90.

63. Ramalingam SS, Maitland ML, Frankel P, et al.

Cite this article as: Sun J, Zhao J, Yang Z, Zhou Z, Lu P. Identification of gene signatures and potential therapeutic targets for acquired chemotherapy resistance in gastric cancer patients. J Gastrointest Oncol 2021;12(2):407-422. doi: 10.21037/ jgo-21-81
Carboplatin and Paclitaxel in combination with either vorinostat or placebo for first-line therapy of advanced non-small-cell lung cancer. J Clin Oncol 2010;28:56-62.

64. Vasan N, Baselga J, Hyman DM. A view on drug resistance in cancer. Nature 2019;575:299-309.

(English Language Editor: C. Betlazar-Maseh) 